\title{
Comparative randomised trial of high and conventional doses of praziquantel in the treatment of schistosomiasis mansoni
}

\author{
Leonardo C de Queiroz', Sandra C Drummond², Maria Laura M de Matos ${ }^{3}$, Mariana BS Paiva ${ }^{3}$, \\ Thaís S Batista ${ }^{3}$, Ahraby ZM Kansaon ${ }^{3}$, Carlos Maurício de F Antunes ${ }^{3}$, \\ José Roberto Lambertucci ${ }^{3} /+$
}

${ }^{1}$ Serviço de Radiologia ${ }^{3}$ Programa de Pós-Graduação em Ciências da Saúde: Infectologia e Medicina Tropical, Hospital das Clínicas, Faculdade de Medicina, Universidade Federal de Minas Gerais, Belo Horizonte, MG, Brasil ${ }^{2}$ Secretaria de Estado de Saúde, Belo Horizonte, MG, Brasil

The efficacy of oral praziquantel in the treatment of schistosomiasis has been considered low by most public health institutions. In this paper, we compared the efficacy of two dosages of praziquantel ( $80 \mathrm{mg} / \mathrm{kg} \mathrm{vs.} 50 \mathrm{mg} / \mathrm{kg}$ ) in patients with chronic schistosomiasis mansoni. Two hundred eighty-eight patients with schistosomiasis from a community in Brazil were randomly divided into two groups: 145 patients (Group 1) received $80 \mathrm{mg} / \mathrm{kg}$ body weight of oral praziquantel divided in two equal doses with 1 h interval and 143 patients (Group 2) received $50 \mathrm{mg} / \mathrm{kg}$ body weight of oral praziquantel. To keep the study masked, patients in Group 2 received placebo 1 h after the first dose. All patients were subjected to clinical and ultrasonographic examination. Cure assessment was performed by repeating two stool examinations, by a quantitative method, at 30, 90 and 180 days after treatment. The morbidity of schistosomiasis was low, with a few cases of light periportal thickening and 16 cases of mild splenomegaly. The cure rates were $89.7 \%$ for Group 1 and $83.9 \%$ for Group 2. There was no difference in the efficacy of both therapeutic dosages of praziquantel assayed. The adverse reactions were more frequent with higher dosage.

Key words: schistosomiasis mansoni - schistosomiasis - praziquantel - treatment - therapeutic efficacy - adverse reactions

From 1976-2003, 12 million subjects were treated for schistosomiasis by the Brazilian Program of Schistosomiasis Control (WHO 1985, Amaral et al. 2006). For many years, oxamniquine, in a single oral dose, was the drug of choice (Lambertucci et al. 1982, 2000, Lambertucci 1993). Presently, praziquantel has been elected the drug of first choice for the treatment of schistosomiasis in Brazil. Treatment costs decreased sharply after the country started its production.

The efficacy of oral praziquantel in the treatment of schistosomiasis has been considered low by most public health institutions of other countries (Doenhoff et al. 2002, Raso et al. 2004, Botros et al. 2005). For the usual dosages in Brazil $(60 \mathrm{mg} / \mathrm{kg}$ body weight for children and $50 \mathrm{mg} / \mathrm{kg}$ for adults), the cure rates varied from 50 $90 \%$, depending on the criteria of cure used by different investigators (Katz \& Rocha 1982, Prata et al. 1982, da Cunha et al. 1987).

A series of alternative regimens has been proposed to improve the efficacy of praziquantel. The combined use of two schistosomicides (Xiao \& Catto 1989, Utzinger et al. 2003), prolongation of treatment time (da Cunha et al. 1987) and an increase in dosages have been suggested. In contrast, some authors have noticed that adverse reactions caused by praziquantel were decreasing cooperation

+ Corresponding author: lamber@uai.com.br

Received 9 January 2009

Accepted 18 December 2009 in some communities when re-treatment for therapeutic failures was necessary. Therefore, researchers suggested that clinical trials with lower dosages would be desirable.

In this paper, we compared the efficacy of two dosages of praziquantel ( $80 \mathrm{mg} / \mathrm{kg}$ vs. $50 \mathrm{mg} / \mathrm{kg}$ body weight) in patients with chronic schistosomiasis mansoni.

\section{PATIENTS, MATERIALS AND METHODS}

Patients - In 2002, all individuals over 13 years of age with a positive stool sample for Schistosoma mansoni infection in Chonin (a district of Governador Valadares, Minas Gerais, Brazil, population of 1,875 inhabitants and prevalence of schistosomiasis of $22.5 \%$ ) were invited to be treated with praziquantel in a randomised trial. All subjects were given two parasitological stool examinations ( 2 slides per stool sample) by the quantitative Kato-Katz technique (Katz et al. 1979). Among the $422 \mathrm{~S}$. mansoni infected inhabitants, $306(72.5 \%)$ ranging from 13-70 years of age, volunteered to participate in the present investigation. All those admitted in the study signed an informed consent for participation.

Criteria for exclusion from the study included pregnancy, cardiomyopathies and chronic liver and renal diseases; however, no participants were excluded.

Three hundred six subjects were randomly assigned into two groups using small blocks to achieve balance between them. Group 1 included 156 patients who received $80 \mathrm{mg} / \mathrm{kg}$ body weight of oral praziquantel, divided in two equal doses with a $1 \mathrm{~h}$ interval between doses. Group 2 included 150 volunteers who received $50 \mathrm{mg} / \mathrm{kg}$ body weight of oral praziquantel plus a similar tablet of placebo $1 \mathrm{~h}$ after the first dose to keep the study masked. 
Clinical examination - Physical examination was conducted by a member of the research team (JRL). Abdominal palpation was performed in patients in dorsal decubitus during deep breathing. Liver and spleen were sought below the costal margins and when palpable their lengths were measured.

Ultrasound - Sonographic examination was conducted by a radiologist trained in the application of the Niamey protocol. A GE Logic Book portable ultrasound was used with a $2.5-5-\mathrm{MHz}$ polifrequencial convex transducer, which allows storage of raw data in Dicom format for future re-evaluation. Subjects were allocated into four groups according to ultrasound classification: (i) no fibrosis, including those classified as World Health Organization pattern A, (ii) light fibrosis, comprising those classified as C, D or Dc, (iii) moderate fibrosis, with those classified as E or Ec and (iv) intense fibrosis, patients classified as F (Lambertucci et al. 2008).

End point - Cure was assessed by repeating two stool examinations with two slides each on 30,90 and 180 days after treatment.

Adverse reactions - In the first $2 \mathrm{~h}$, adverse reactions were observed by the attending physician. Up to $48 \mathrm{~h}$ after treatment, they were assessed by health agents at patients' homes following a structured protocol, which included previously described adverse reactions to praziquantel: headache, weakness, dizziness, abdominal pain, nausea, vomiting, fever, urticaria, anorexia, sweating and bitter taste (Katz \& Rocha 1982, Kabatereine et al. 2003).

Ethical aspects - This study was approved by the Ethical Review Board of the Universidade Federal de Minas Gerais. To be included in the clinical trial, all patients were asked to sign an informed consent. The consent for minors was obtained from their parents or responsible adult caring for the child. All procedures followed the recommendations contained in the Helsinki Protocol (World Medical Association 1975, revised in 1983).

Statistical analysis - A databank was built using EpiData 3.1 software. The data were analysed using EpiInfo 3.2 and SPSS 12.0 statistical packages. Initial analysis compared the characteristics of Groups 1 and 2 in order to evaluate the random allocation. Praziquantel efficacy and adverse reactions were investigated using the intention to treat principle. Survival analysis was used to compare praziquantel efficacy between the two groups (Kaplan-Meier test).

\section{RESULTS}

Clinical findings - One patient had intense liver fibrosis and portal hypertension with a collateral left gastric vein; 16 had mild splenomegaly without significant periportal thickening; one was splenectomised. The remaining patients were classified as having hepatointestinal schistosomiasis.

Regarding demographic and clinical characteristics, no significant differences were observed between the two study groups (Table I). In Group 1, the mean number of eggs/gram of faeces was 137.5, compared with 203.9 in Group $2(\mathrm{p}=0.103)$.
Cure was assessed by repeating stool examinations ( 2 samples, 2 slides each) on 30, 90 and 180 days after treatment. However, not all participants complied with the evaluation schedule; the number of examinations after treatment is depicted in Table II. Eighteen subjects (11 from Group 1 and 7 from Group 2) were lost at follow-up.

The overall efficacy of praziquantel was $86.8 \%$; in Groups 1 and 2, the observed efficacy was $89.7 \%$ and $83.9 \%$, respectively (intention to treat analysis). This difference was not statistically significant. Additionally, no significant statistical difference was observed upon comparing the cumulative survival curves between the two groups (Kaplan-Meier, $p=0.543$; Mantel-Cox). For Groups 1 and 2 respectively, a total of 92 and 106 days passed before an $S$. mansoni stool sample tested positive.

Adverse reactions - Upon evaluation $2 \mathrm{~h}$ after treatment, 169 patients (58.6\%) reported at least one adverse reaction, with no statistical difference between Groups 1 and $2(\mathrm{p}=0.328)$. Forty-eight hours after treatment, 247 patients were questioned about adverse reactions. In Group 1, 111 patients $(57.2 \%)$ reported at least one adverse reaction; in Group 2, 83 patients (42.8\%) reported adverse events, revealing a significant statistical difference $(p=0.003)$.

Adverse reactions reported by patients in both groups were, in order of frequency, dizziness, nausea, headache, vomiting, sweating and anorexia.

The most frequent side effects observed $2 \mathrm{~h}$ after treatment were nausea and dizziness. Dizziness, weakness, nausea and somnolence were most frequently described $48 \mathrm{~h}$ after treatment (Tables III, IV).

TABLE I

Baseline characteristics of the study population

\begin{tabular}{|c|c|c|c|c|c|}
\hline \multirow[t]{2}{*}{ Characteristics } & \multicolumn{2}{|c|}{ Group 1} & \multicolumn{2}{|c|}{ Group 2} & \multirow[t]{2}{*}{$\mathrm{p}$} \\
\hline & Male & Female & Male & Female & \\
\hline Gender (n) & 87 & 58 & 83 & 60 & 0.735 \\
\hline Age (mean) & \multicolumn{2}{|c|}{31.7} & \multicolumn{2}{|c|}{31.2} & 0.725 \\
\hline Weight (mean) & \multicolumn{2}{|c|}{64.4} & \multicolumn{2}{|c|}{64.6} & 0.924 \\
\hline Height (mean) & \multicolumn{2}{|c|}{165.5} & \multicolumn{2}{|c|}{165.2} & 0.748 \\
\hline
\end{tabular}

\section{TABLE II}

Number of stool examinations for Groups 1 and 2 after treatment in Chonin, Minas Gerais, Brazil, 2002

\begin{tabular}{cc}
\hline Number of examinations & $\begin{array}{c}\text { Patients } \\
\mathrm{n}(\%)\end{array}$ \\
\hline 1 & $86(29.9)$ \\
2 & $106(36.8)$ \\
3 & $96(33.3)$
\end{tabular}


TABLE III

Adverse reactions $2 \mathrm{~h}$ after treatment in Chonin, Minas Gerais, Brazil, 2002

\begin{tabular}{lcccc}
\hline Adverse reactions & Group 1 & Group 2 & Total & $\mathrm{p}$ \\
\hline Bitter taste & 65 & 73 & 138 & 0.291 \\
Dizziness & 23 & 25 & 48 & 0.712 \\
Abdominal pain & 10 & 10 & 20 & 0.974 \\
Nausea & 14 & 6 & 20 & 0.068 \\
Headache & 6 & 6 & 12 & 0.980 \\
Somnolence & 6 & 3 & 9 & 0.320 \\
Weakness & 4 & 3 & 7 & 0.716 \\
Vomiting & 3 & 1 & 4 & 0.307 \\
\hline Total & 81 & 88 & 169 & 0.328 \\
\hline
\end{tabular}

TABLE IV

Adverse reactions $48 \mathrm{~h}$ after treatment in Chonin, Minas Gerais, Brazil, 2002

\begin{tabular}{lcccc}
\hline Adverse reactions & Group 1 & Group 2 & Total & $\mathrm{p}$ \\
\hline Dizziness & 74 & 52 & 126 & 0.037 \\
Weakness & 58 & 47 & 105 & 0.415 \\
Bitter taste & 58 & 40 & 98 & 0.076 \\
Nausea & 49 & 26 & 75 & 0.006 \\
Headache & 46 & 28 & 74 & 0.041 \\
Vomiting & 44 & 14 & 58 & 0.000 \\
Diarrhea & 28 & 18 & 46 & 0.193 \\
Anorexia & 28 & 14 & 42 & 0.040 \\
Sweating & 29 & 13 & 42 & 0.017 \\
Abdominal pain & 17 & 17 & 34 & 0.780 \\
Fever & 12 & 10 & 22 & 0.820 \\
Somnolence & 7 & 7 & 14 & 0.864 \\
Myalgia & 6 & 6 & 12 & 0.874 \\
Urticaria & 5 & 3 & 8 & 0.554 \\
\hline Total & 111 & 83 & 194 & 0.003 \\
\hline
\end{tabular}

\section{DISCUSSION}

In this study, no difference was observed in the efficacy of praziquantel dosages $(80 \mathrm{mg} / \mathrm{kg}$ vs. $50 \mathrm{mg}$ / $\mathrm{kg}$ body weight) assayed. However, adverse reactions to treatment were more frequent in the higher dosage group. The morbidity of schistosomiasis was low, with a few cases of light periportal thickening and 16 cases with mild splenomegaly. The majority of subjects were classified as presenting hepatointestinal schistosomiasis (Lambertucci et al. 1987, 2001, Drummond et al. 2006).

The design of our study was based on the assumption that oral praziquantel taken in higher dosages, equally divided into intervals of $2 \mathrm{~h}$, would improve efficacy and decrease adverse reactions. Praziquantel has a short half life, thus decreasing the drug plasma level (Leopold et al. 1978) would decrease the frequency of adverse effects. The $2 \mathrm{~h}$ interval between drug intake proved difficult to manage in field conditions. As a result, the doses were administered with a $1 \mathrm{~h}$ interval between doses.

As mentioned previously, the reported efficacy of schistosomicides is dependent on the criteria of cure used by the different investigators (Katz \& Rocha 1982, Prata et al. 1982, da Cunha et al. 1987). Additionally, in areas with low egg counts, the quantitative stool examination has drawbacks because it has a lower detection limit of 24 eggs per gram of faeces (Kato-Katz method). For example, some authors prefer rectal biopsy for cure control (da Cunha et al. 1987), but this method is difficult to perform in field condition. Therefore, most investigators defend the use of repeated stool examinations (Rabello et al. 1992). Increasing the number of stool examinations will increase the probability of finding positive samples and consequently decrease the putative efficacy of the drug. In our study, for example, about $30 \%$ of patients had only one sample examined after treatment. Even considering that there was no difference in the number of stool examinations between groups, a larger number of tests would increase the overall sensitivity, increasing the possibility that a statistical difference between treatments would be detected.

The adverse reactions were more frequent in the higher dosage group when assessed $48 \mathrm{~h}$ after treatment. Although adverse effects have been described more frequently with higher dosage, they did not differ from those reported in the literature for praziquantel (Prata et al. 1982, Coutinho et al. 1984, Kabatereine et al. 2003).

In conclusion, there was no advantage in the use of praziquantel at a dosage of $80 \mathrm{mg} / \mathrm{kg}$ body weight in regard to efficacy when compared to $50 \mathrm{mg} / \mathrm{kg}$. In addition, it was disadvantageous in terms of adverse reactions.

\section{REFERENCES}

Amaral RS, Tauil PL, Lima DD, Engels D 2006. An analysis of the impact of the Schistosomiasis Control Programme in Brazil. Mem Inst Oswaldo Cruz 101 (Suppl. I): 79-85.

Botros S, Sayed H, Amer N, El-Ghannam M, Bennett JL, Day TA 2005. Current status of sensitivity to praziquantel in a focus of potential drug resistance in Egypt. Int J Parasitol 35: 787-791.

Coutinho AD, Domingues AL, Florêncio JN, Almeida ST 1984. Treatment of hepatosplenic schistosomiasis mansoni with praziquantel. Rev Inst Med Trop Sao Paulo 26: 38-50.

da Cunha AS, Cançado JR, de Rezende GL 1987. Therapeutical evaluation of different dose regimens of praziquantel in schistosomiasis mansoni, based on the quantitative oogram technique. Rev Inst Med Trop Sao Paulo 29: 295-304.

Doenhoff MJ, Kusel JR, Coles GC, Cioli D 2002. Resistance of Schistosoma mansoni to praziquantel: is there a problem? Trans $R$ Soc Trop Med Hyg 96: 465-469.

Drummond SC, Silva LCS, Amaral RS, Sousa-Pereira SR, Antunes CM, Lambertucci JR 2006. Morbidity of schistosomiasis mansoni in the state of Minas Gerais, Brazil. Mem Inst Oswaldo Cruz 101 (Suppl. I): 37- 44.

Kabatereine NB, Kemijumbi J, Ouma JH, Sturrock RF, Butterworth AE, Madsen H, Ornbjerg N, Dunne DW, Vennnervald BJ 2003. 
Efficacy and side effects of praziquantel treatment in a highly endemic Schistosoma mansoni focus at Lake Albert, Uganda. Trans R Soc Trop Med Hyg 97: 599-603.

Katz N, Rocha RS 1982. Double-blind clinical trial comparing praziquantel with oxamniquine in schistosomiasis mansoni. Rev Inst Med Trop Sao Paulo 24: 310-314.

Katz N, Rocha RS, Chaves A 1979. Preliminary trials with praziquantel in human infections due to Schistosoma mansoni. Bull World Health Organ 57: 781-785.

Lambertucci JR 1993. Schistosoma mansoni: pathological and clinical aspects. In P Jordan, G Webbe, RF Sturrock (eds.), Human schistosomiasis, Cab International, Wallingford, p. 195-225.

Lambertucci JR, Cota GF, Pinto-Silva RA, Serufo JC, GerspacherLara R, Drummond SC, Antunes CM, Nobre V, Rayes A 2001. Hepatosplenic schistosomiasis in field-based studies: a combined clinical and sonographic definition. Mem Inst Oswaldo Cruz 96 (Suppl.): 147-150.

Lambertucci JR, dos Santos Silva LC, Andrade LM, de Queiroz LC, Carvalho VT, Voieta I, Antunes CM 2008. Imaging techniques in the evaluation of morbidity in schistosomiasis mansoni. Acta Trop 108: 209-217.

Lambertucci JR, Greco DB, Pedroso ER, da Costa Rocha MO, Salazar HM, de Lima DP 1982. A double blind trial with oxamniquine in chronic schistosomiasis mansoni. Trans R Soc Trop Med Hyg 76: 751-755.

Lambertucci JR, Rocha RS, Carvalho OS, Katz N 1987. A esquistossomose mansoni em Minas Gerais. Rev Soc Bras Med Trop 20: 47-52.
Lambertucci JR, Serufo JC, Gerspacher-Lara R, Rayes AA, Teixeira R, Nobre V, Antunes CM. 2000. Schistosoma mansoni: assessment of morbidity before and after control. Acta Trop 77: 101-109.

Leopold G, Ungethüm W, Groll E, Diekmann HW, Nowak H, Wegner DH 1978. Clinical pharmacology in normal volunteers of praziquantel, a new drug against schistosomes and cestodes. An example of a complex study covering both tolerance and pharmacokinetics. Eur J Clin Pharmacol 14: 281-291.

Prata A, Castro N, Silva AE, Paiva M, Macêdo V, Junqueira LF Jr 1982. Praziquantel in the treatment of schistosomiasis mansoni. Rev Inst Med Trop Sao Paulo 24: 95-103.

Rabello AL, Rocha RS, de Oliveira JP, Katz N, Lambertucci JR 1992. Stool examination and rectal biopsy in the diagnosis and evaluation of therapy of schistosomiasis mansoni. Rev Inst Med Trop Sao Paulo 34: 601-608.

Raso G, N'Goran EK, Toty A, Luginbühl A, Adjoua CA, Tian-Bi NT, Bogoch II, Vounatsou P, Tanner M, Utzinger J 2004. Efficacy and side effects of praziquantel against Schistosoma mansoni in a community of western Côte d'Ivoire. Trans R Soc Trop Med Hyg 98: 18-27.

Utzinger J, Keiser J, Shuhua X, Tanner M, Singer BH 2003. Combination chemotherapy of schistosomiasis in laboratory studies and clinical trials. Antimicrob Agents Chemother 47: 1487-1495.

WHO - World Health Organization 1985. The control of schistosomiasis. Report of a WHO Expert Committee. World Health Organ Tech Rep Ser 728: 1-113.

Xiao SH, Catto BA 1989. In vitro and in vivo studies of the effect of artemether on Schistosoma mansoni. Antimicrob Agents Chemother 33: 1557-1562. 